Characteristic morphological changes to the endothelium of abdominal and thoracic arteries (Fig. 1) were projections of foot processes into the lumen and an increase in the number of pinocytotic vessels relative to controls. This was similar to the changes observed by STILL and ScotT [2] in capillary endothelia. In one animal intra-endothelial vacuoles contained electron dense particles (Fig. 2) of a diameter similar in size to Coli endotoxin [3]. The lumen lining of the basilar artery (Fig. 3) was often covered by a layer of granular material resembling the hyalin deposits described by FisHer et al. [4] in human arterioles following hypertension, and believed to be of fibrinoid origin. A detailed study of this phenomenon is presently in progress.

Received February 14, 1967

[1] Mc Kay, D., and S. Shapiro: J. exp. Med. 107, 353 (1958). [2] StilL, W., and G. Scotr: Exp. Mol. Pathol. 5, 118 (1966). [3] Betr, H., A. Braude, and C. Brinton: Ann. N. Y. Acad. Sci. 133, 450 (1966). - [4] Fisher, E., E. Perez-Stable, and V. PARDo: Lab. Invest. 15, 1409 (1966).

\section{Subjective Vertical as a Function of Body Position and Gravity Magnitude}

\section{H. Schöne, D. E. PARkeR ${ }^{\star}$, and H. G. Mortag}

\section{Max-Planck-Institut für Verhaltensphysiologie, Seewiesen}

Perception of spatial coordinates can be related to the functioning of the statolith-organs [1-5]. Previous observations indicate that the subjective height of horizon changes in proportion to the shearing force acting on the utricular maculae over a limited range of forward-backward tilted body positions $[4,5]$.

In our recent experiments we investigated the subjective vertical (SV). The subject (S) was tighly restrained in a bed; the head was bent backwards so that in the zero degree position (head vertex up) the utricular maculae lay in approximately the horizontal plane. Head position was maintained

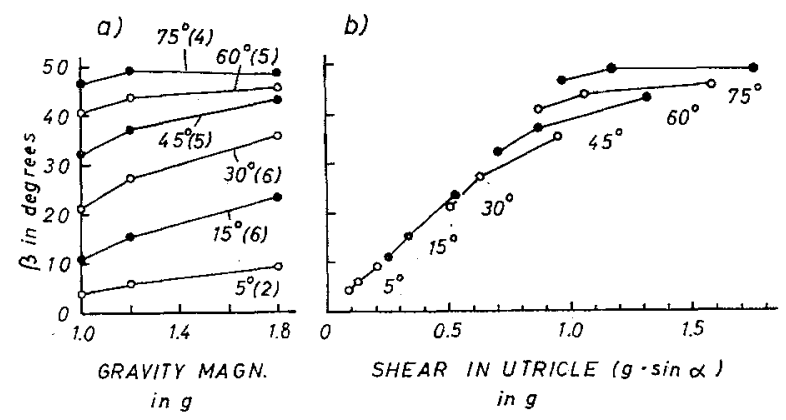

Fig. 1. SV $(\beta)$ as a function of a gravity magnitude and $b$ shearing force in the utricles at different $\alpha$; number of $\mathrm{Ss}$ in brackets

by a bite board. The bed, which could be rotated through $360^{\circ}$, was placed in the gondola of a centrifuge. During centrifugation the magnitude (g) of gravity (resultant vector of centrifugal and gravity acceleration) changed, but the direction relative to $S$ remained constant.

Ss were placed in laterally tilted positions of $5,15,30,45,60$ and $75^{\circ}$ of angle between head vertex-base axis and resultant vector $(\alpha)$. Each position was investigated at force levels of $1.0,1.2$ and $1.8 \mathrm{~g}$. $\mathrm{S}$ was required to determine the SV by manipulating a luminous line at $15 \mathrm{sec}$ intervals for $4 \mathrm{~min}$; the position of the line was recorded automatically. The average of the last minute was taken as SV for the particular run. SV was specified as the angle between luminous line and head vertex-base axis $(\beta)$.

As Fig. 1 a shows, the slopes of the curves increase with $\alpha$ up to $30^{\circ}$, whereas at higher $\alpha$ they approach zero. In order to relate the data to utricular function, all points were replotted in terms of $\beta$ as a function of the utricular shearing force. Fig. $1 \mathrm{~b}$ illustrates a linear relationship between $\beta$ and utricular shear over a range of zero to $0.8 \mathrm{~g}$. Beyond $0.8 \mathrm{~g}$ of shear there are discontinuities and deviations from linearity and after $1 \mathrm{~g}$ the curves appear flat. We did not succeed in expressing the data in terms of a tangent or arcus tangent equation (cp. [1]).
The result of the flattening out may be interpreted as a "saturation" effect. At $1 \mathrm{~g}$ shearing a physiological limit is reached, increased stimulus intensity does not produce a shift in SV. Further explanation suggests interaction between the utricles and other gravity receptors, perhaps the saccules.

Received January 20, 1967

* Dept. Psychol., Oxford, Ohio. Supported by Special Research Fellowship MH-12,310, United States Public Health Service.

[1] Correta, M. J., W. C. Hrxon, and J. I. Niven: Joint Rep. NAMI-951, NASA order R-93, Pensacola, Fla. Nav Aerospace Med. Inst. 1965. - [2] MeIRY, J. L : Doctoral Diss. T-65-1, Mass. Inst. Technol. 1965. [3] Mrluer, E. F., and A. Graybiel: Joint Rep. NSAM-901, NASA order R-47. Pensacola, Fla.: Nav. Aerosp. Med. Inst. 1964. - [4] SCHÖNE, H.: Z. vergleich. Physiol. 46, 57 (1962). $\rightarrow[5]$ Schöne, H.: Aerospace Med. 35, 764 (1964).

\section{Inversion of the Effect of Increased Gravity on the Subjective Vertical}

H. SchöNe and D. E. PARKER*

Max-Planck-Institut für Verhaltensphysiologie, Seewiesen

The Subjective Vertical (SV) can be related to the shearing force acting on the utricular maculae $[1-4]$. It changes in linear proportion with the shear over a range of zero to $0.8 \mathrm{~g}$; however, discontinuities and a "saturation" effect are produced when the shearing force passes $1.0 \mathrm{~g}$, which is equivalent to a lateral tilt of $90^{\circ}$ under normal gravity conditions $[4]$.

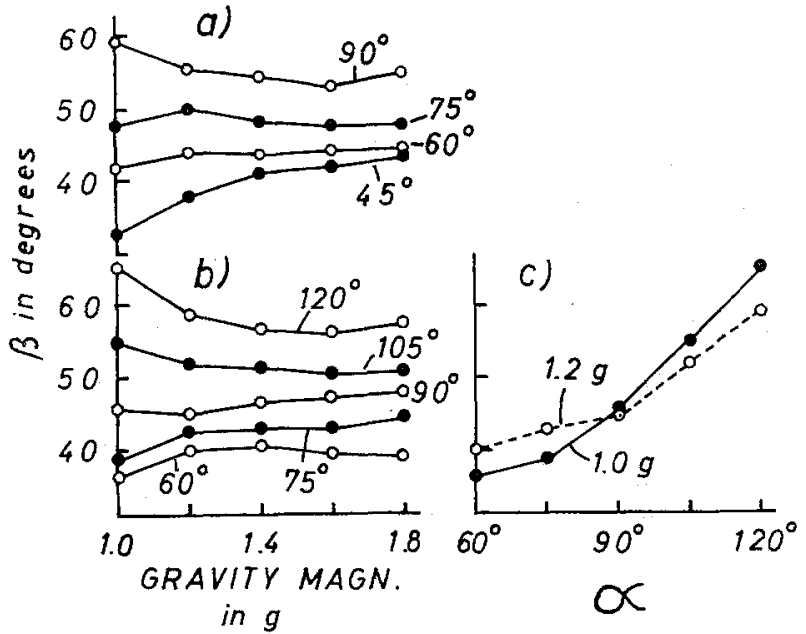

Fig. $1 \mathrm{a}-\mathrm{C}$. SV $(\rho)$ as a function of gravity magnitude at different $\alpha$; $a$ average of two $\mathrm{Ss}(B, P) ;-b$ average of two Ss $(M, S) ;-c \mathrm{SV}$ as a function of $\alpha$, same data as in $b$

We tried to explore the effect of increased gravity fields during lateral body tilts in the range of $90^{\circ}$. Two resp. four subjects (Ss) were studied in body positions of $45,60,75$, 90,105 and $120^{\circ}$ (angle $\alpha$ ) at force levels of $1.0,1.2,1.4,1.6$ and $1.8 \mathrm{~g}$. SV was recorded as the angle between a luminous line and the head vertex-base axis (angle $\beta$ ). Methodological details are given elsewhere $[4]$.

In the body positions below $90^{\circ}$ an increase in $\mathrm{g}$ produced an increase in $\beta$ up to the saturation level (Fig. $1 \mathrm{a}, \mathrm{b}$ ), which is in accordance with former results [4]. For the body positions above $90^{\circ}$ increasing $g$ produced an opposite effect: decrease rather than increase in $\beta$. As before, a saturation effect was noted. If the data are replotted in terms of $\beta$ as a function of $\alpha$, the inversion effect is clearly visible (Fig. $1 \mathrm{c}$ ): the curves for $1.0 \mathrm{~g}$ and $1.2 \mathrm{~g}$ cross each other near the $90^{\circ}$ point.

The inversion phenomenon may be interpreted as a result of a sign change for some signal variable associated with the system leading from sensory input to perceptual output. It should occur at a relatively high level because no inversion effect of this kind has been observed in the counterrolling of the eye [2]. The interpretation may be expressed in terms of the shearing hypothesis. Raising of gravity magnitude will produce increases in utricular shearing and consequently also increases of the angle of SV; this is correct for positions under $90^{\circ}$ as 